\title{
A Survey on Estimates for the Differences of Positive Linear Operators
}

\author{
ANA-MARIA ACU, SEVER HODIŞ, AND IOAN RAŞA
}

ABSTRACT. We survey some results concerning differences of positive linear operators from Approximation Theory, and present some new results in this direction.

Keywords: Positive linear operators, Inverses of operators, Moduli of smoothness, Estimations of differences of operators

2010 Mathematics Subject Classification: 41A25, 41A36.

\section{INTRODUCTION}

In the recent years the differences of positive linear operators have been investigated from several points of view. The aim of this paper is to survey some of the known results and to present some new ones.

In Section 2 we recall the definitions of some classical operators used in Approximation Theory and some general results concerning their differences. These results are illustrated with the corresponding classical operators. Section 3 is devoted to the differences of the operators constructed with the same fundamental functions, only the functionals in front of them being different. In Section 4 we consider differences of operators from the family $U_{n}^{\rho}$. The case of operators on unbounded intervals is discussed in Section 5. Operators and their derivatives are considered in Section 6, while Section 7 is devoted to discrete operators versus integral operators. Finally, new results concerning operators and their inverses are presented in Section 8.

Throughout the paper $C[0,1]$ stands for the space of all continuous real-valued functions, endowed with the supremum norm and usual ordering.

\section{SOME GENERAL RESULTS}

The classical Bernstein operators introduced by Bernstein [5] in order to prove Weierstrass's fundamental theorem are given by

$$
\begin{gathered}
B_{n}: C[0,1] \rightarrow C[0,1], B_{n}(f ; x)=\sum_{k=0}^{n} f\left(\frac{k}{n}\right) p_{n, k}(x), \text { where } \\
p_{n, k}(x)=\left(\begin{array}{c}
n \\
k
\end{array}\right) x^{k}(1-x)^{n-k}, x \in[0,1] .
\end{gathered}
$$

Received: 4 November 2018; In revised form: 6 November 2018; Accepted: 7 November 2018

*Corresponding author: A. M. Acu; anamaria.acu@ulbsibiu.ro

DOI: $10.33205 / \mathrm{cma} .478408$ 
Let $n=1,2,3, \ldots$ and $f \in C[0,1]$. The Beta-type operators $\overline{\mathbb{B}}_{n}$ were introduced by Lupaş in his German thesis [19] as follows

$$
\overline{\mathbb{B}}_{n}(f ; x):=\left\{\begin{array}{l}
f(0), x=0, \\
\frac{1}{B(n x, n-n x)} \int_{0}^{1} t^{n x-1}(1-t)^{n-1-n x} f(t) d t, 0<x<1, \\
f(1), x=1,
\end{array}\right.
$$

where $B(\cdot, \cdot)$ is the Euler's Beta function.

The genuine Bernstein-Durrmeyer operators were introduced by Chen [6] and Goodman and Sharma [13] as a composition of Bernstein operators and Beta operators, namely $U_{n}:=$ $B_{n} \circ \overline{\mathbb{B}}_{n}$. These are given in explicit form by

$$
U_{n}(f ; x)=(1-x)^{n} f(0)+x^{n} f(1)+(n-1) \sum_{k=1}^{n-1}\left(\int_{0}^{1} f(t) p_{n-2, k-1}(t) d t\right) p_{n, k}(x), f \in C[0,1] .
$$

Denote by $S_{n}:=\overline{\mathbb{B}}_{n} \circ B_{n}$ the Stancu-type operator investigated in [20] and defined as

$$
S_{n}(f ; x)=\frac{1}{(n)_{n}} \sum_{k=0}^{n}\left(\begin{array}{l}
n \\
k
\end{array}\right)(n x)_{k}(n-n x)_{k} f\left(\frac{k}{n}\right),
$$

where the rising factorial $(x)_{k}$ is given by $(x)_{k}=x(x+1)(x+2) \ldots(x+k-1)$ with $(x)_{0}=1$.

Let

$$
\omega_{k}(f ; h):=\sup \left\{\left|\sum_{j=0}^{k}(-1)^{k-j}\left(\begin{array}{l}
k \\
j
\end{array}\right) f(x+j \delta)\right|:|\delta| \leq h, x, x+j \delta \in I\right\}
$$

be the classical $k^{t h}$ order modulus of smoothness given for a compact interval $I$ and $h \in \mathbb{R}_{+}$ and $\tilde{\omega}$ is the least concave majorant of the first order modulus $\omega_{1}$ given by

$$
\tilde{\omega}(f ; t)=\sup \left\{\frac{(t-x) \omega_{1}(f ; y)+(y-t) \omega_{1}(f ; x)}{y-x}: 0 \leq x \leq t \leq y \leq b-a, x \neq y\right\} .
$$

The study of differences of certain positive and linear operators has as starting point the problem proposed by Lupaş [18], namely the question raised by him was to give an estimate for the difference $U_{n}-S_{n}$. A solution for the problem proposed by Lupaş in a more general case was given by Gonska et al. [10] and we recall their results below:

Theorem 2.1. [10] Let $A, B: C[0,1] \rightarrow C[0,1]$ be positive linear operators such that

$$
(A-B)\left(\left(e_{1}-x\right)^{i} ; x\right)=0 \text { for } i=0,1,2,3 \text { and } x \in[0,1] \text {. }
$$

Then for $f \in C^{3}[0,1]$ there holds

$$
|(A-B)(f ; x)| \leq \frac{1}{6}(A+B)\left(\left|e_{1}-x\right|^{3} ; x\right) \tilde{\omega}\left(f^{\prime \prime \prime} ; \frac{1}{4} \frac{(A+B)\left(\left(e_{1}-x\right)^{4} ; x\right)}{(A+B)\left(\left|e_{1}-x\right|^{3} ; x\right)}\right) .
$$

Theorem 2.2. [10] If $A$ and $B$ are given as in Theorem 2.1, satisfying $A e_{0}=B e_{0}=e_{0}$, then for all $f \in C[0,1], x \in[0,1]$ we have

$$
|(A-B)(f ; x)| \leq c_{1} \omega_{4}\left(f ; \sqrt[4]{\frac{1}{2}(A+B)\left(\left(e_{1}-x\right)^{4} ; x\right)}\right) .
$$

Here $c_{1}$ is an absolute constant independent of $f, x, A$ and $B$.

Using the above result the following solution to Lupaş' problem was given in [10]: 
Proposition 2.1. The Beta operators and the Bernstein operators verify

$$
\left|\left(U_{n}-S_{n}\right)(f ; x)\right| \leq c_{1} \omega_{4}\left(f ; \sqrt[4]{\frac{3 x(1-x)}{n(n+1)}}\right) .
$$

Here $c_{1}$ is an absolute constant independent of $n, f$ and $x$.

Gonska et al. have continued their research on the differences of positive linear operators in [8]-[9]. The inequality of Theorem 2.1 was extended for a more general case as follows:

Theorem 2.3. [9] Let $A, B: C[0,1] \rightarrow C[0,1]$ be positive operators such that

$$
(A-B)\left(\left(e_{1}-x\right)^{i} ; x\right)=0 \text { for } i=0,1, \ldots, n \text { and } x \in[0,1] .
$$

Then for $f \in C^{n}[0,1]$ there holds

$$
|(A-B)(f ; x)| \leq \frac{1}{n !}(A+B)\left(\left|e_{1}-x\right|^{n} ; x\right) \tilde{\omega}\left(f^{(n)} ; \frac{1}{n+1} \frac{(A+B)\left(\left|e_{1}-x\right|^{n+1} ; x\right)}{(A+B)\left(\left|e_{1}-x\right|^{n} ; x\right)}\right) .
$$

The result from Theorem 2.2 was extended in [9] as follows:

Theorem 2.4. [9] If $A$ and $B$ are given as in Theorem 2.3, satisfying $A e_{0}=B e_{0}=e_{0}$, then for all $f \in C[0,1], x \in[0,1]$ we have

$$
|(A-B)(f ; x)| \leq c_{1} \omega_{n+1}\left(f ; \sqrt[n+1]{\frac{1}{2}(A+B)\left(\left|e_{1}-x\right|^{n+1} ; x\right)}\right) .
$$

Here $c_{1}$ is an absolute constant independent of $f, x, A$ and $B$.

In [8] Gonska, Piţul and Raşa applied the above results for some known positive linear operators as the Bernstein operators $B_{n}$, the Beta operators $\overline{\mathbb{B}}_{n}$, the genuine Bernstein-Durrmeyer operators $U_{n}=B_{n} \circ \overline{\mathbb{B}}_{n}$ and the composition of two Bernstein operators $D_{n}=B_{n} \circ B_{n+1}$.

Proposition 2.2. [9] The Bernstein operators and the Beta-type operators verify the following relations:

$$
\begin{aligned}
i)\left|\left(B_{n+1}-\overline{\mathbb{B}}_{n}\right)(f ; x)\right| & \leq \frac{x(1-x)}{n+1} \tilde{\omega}\left(f^{\prime \prime} ; \sqrt{\frac{(n+1)(6 n x(1-x)+7)}{18 n^{2}}}\right), f \in C^{2}[0,1] \\
& \leq \frac{x(1-x)}{3 n \sqrt{n+1}} \sqrt{\frac{6 n x(1-x)+7}{2 n}}\left\|f^{\prime \prime \prime}\right\|, f \in C^{3}[0,1] ; \\
i i)\left|\left(B_{n+1}-\overline{\mathbb{B}}_{n}\right)(f ; x)\right| & \leq c \omega_{3}\left(f ; \sqrt[3]{\frac{1}{2}\left(B_{n+1}+\overline{\mathbb{B}}_{n}\right)\left(\left|e_{1}-x\right|^{3} ; x\right)}\right) \\
& \leq c \omega_{3}\left(f ; \sqrt[6]{\frac{x^{2}(1-x)^{2}}{n^{3}} \cdot \frac{6 n x(1-x)+7}{n}}\right) .
\end{aligned}
$$

Proposition 2.3. [9] The Bernstein operators and the genuine Bernstein-Durrmeyer operators verify the following relation:

$$
\left|\left(B_{n}-U_{n}\right)(f ; x)\right| \leq c \omega_{2}\left(f ; \sqrt{\frac{3 x(1-x)}{2 n}}\right)
$$


Proposition 2.4. [9] The composition of two Bernstein operators $D_{n}=B_{n} \circ B_{n+1}$ and the genuine Bernstein-Durrmeyer operators $U_{n}$ verify the following relations:

$$
\begin{aligned}
i)\left|\left(D_{n}-U_{n}\right)(f ; x)\right| & \leq \frac{2 x(1-x)}{n+1} \tilde{\omega}\left(f^{\prime \prime} ; \sqrt{\frac{(n+1)(8 n x(1-x)+13)}{12 n^{3}}}\right), f \in C^{2}[0,1], \\
& \leq \frac{x(1-x)}{n \sqrt{n+1}} \sqrt{\frac{8 n x(1-x)+13}{3 n}}\left\|f^{\prime \prime \prime}\right\|, f \in C^{3}[0,1] ; \\
i i)\left|\left(D_{n}-U_{n}\right)(f ; x)\right| & \leq c \omega_{3}\left(f ; \sqrt[3]{\frac{1}{2}\left(D_{n}+U_{n}\right)\left(\left|e_{1}-x\right|^{3} ; x\right)}\right) \\
& \leq c \omega_{3}\left(f ; \sqrt[6]{\frac{x^{2}(1-x)^{2}}{(n+1) n^{3}}(24 n x(1-x)+39)}\right) .
\end{aligned}
$$

\section{DiFFERENCES OF POSITIVE LINEAR OPERATORS WITH THE SAME FUNDAMENTAL FUNCTIONS}

The results mentioned in the previous section are based on the fact that $A$ and $B$ have the same moments up to a certain order. The approach presented in this section involves operators constructed with the same "fundamental functions", but with different functionals in front of them (see [2]). So the difference $A-B$ is controlled by the differences of these functionals.

Let $I \subset \mathbb{R}$ be an interval and $E(I)$ a space of real-valued continuous functions on $I$ containing the polynomials. $E_{b}(I)$ will be the space of all $f \in E(I)$ with

$$
\|f\|:=\sup \{|f(x)|: x \in I\}<\infty .
$$

For $i \in \mathbb{N}$ let $e_{i}(x):=x^{i}, x \in I$. Let $F: E(I) \rightarrow \mathbb{R}$ be a positive linear functional such that $F\left(e_{0}\right)=1$. Set $b^{F}:=F\left(e_{1}\right)$ and

$$
\mu_{i}^{F}:=\frac{1}{i !} F\left(e_{1}-b^{F} e_{0}\right)^{i}, i \in \mathbb{N} .
$$

Then $\mu_{0}^{F}=1, \mu_{1}^{F}=0, \mu_{2}^{F}=\frac{1}{2}\left(F\left(e_{2}\right)-\left(b^{F}\right)^{2}\right) \geq 0$.

Lemma 3.1. [2] Let $f \in E(I)$ with $f^{\prime \prime} \in E_{b}(I)$. Then

$$
\left|F(f)-f\left(b^{F}\right)\right| \leq \mu_{2}^{F}\left\|f^{\prime \prime}\right\| .
$$

Lemma 3.2. [2] Let $f \in E(I)$ with $f^{I V} \in E_{b}(I)$. Then

$$
\left|F(f)-f\left(b^{F}\right)-\mu_{2}^{F} f^{\prime \prime}\left(b^{F}\right)-\mu_{3}^{F} f^{\prime \prime \prime}\left(b^{F}\right)\right| \leq \mu_{4}^{F}\left\|f^{I V}\right\| .
$$

Proposition 3.5. [2] Let $I=[0,1], f \in C[0,1], \lambda \geq 2 \sqrt{\mu_{2}^{F}}>0$. Then

$$
\left|F(f)-f\left(b^{F}\right)\right| \leq \frac{3}{2} \omega_{2}\left(f, \frac{\sqrt{\mu_{2}^{F}}}{\lambda}\right)\left(1+\lambda^{2}\right) .
$$

Let $K$ be a set of non-negative integers and $p_{k} \in C(I), p_{k} \geq 0, k \in K$, such that $\sum_{k \in K} p_{k}=e_{0}$. For each $k \in K$ let $F_{k}: E(I) \rightarrow \mathbb{R}$ and $G_{k}: E(I) \rightarrow \mathbb{R}$ be positive linear functionals such that $F_{k}\left(e_{0}\right)=G_{k}\left(e_{0}\right)=1$. Let $D(I)$ be the set of all $f \in E(I)$ for which $\sum_{k \in K} F_{k}(f) p_{k} \in C(I)$ and $\sum_{k \in K} G_{k}(f) p_{k} \in C(I)$ 
Consider the positive linear operators $V: D(I) \rightarrow C(I)$ and $W: D(I) \rightarrow C(I)$ defined, for $f \in D(I)$, by

$$
\begin{array}{r}
V(f ; x):=\sum_{k \in K} F_{k}(f) p_{k}(x) \text { and } W(f ; x):=\sum_{k \in K} G_{k}(f) p_{k}(x) . \\
\text { Denote } \sigma(x):=\sum_{k \in K}\left(\mu_{2}^{F_{k}}+\mu_{2}^{G_{k}}\right) p_{k}(x) \text { and } \delta:=\sup _{k \in K}\left|b^{F_{k}}-b^{G_{k}}\right| .
\end{array}
$$

Theorem 3.5. [2] Let $f \in D(I)$ with $f^{\prime \prime} \in E_{b}(I)$. Then

$$
|(V-W)(f ; x)| \leq\left\|f^{\prime \prime}\right\| \sigma(x)+\omega_{1}(f, \delta) .
$$

Theorem 3.6. [2] Suppose that $b^{F_{k}}=b^{G_{k}}=b_{k}, k \in K$. Let $f \in D(I)$ with $f^{\prime \prime}, f^{\prime \prime \prime}, f^{I V} \in E_{b}(I)$. Then for each $x \in I$,

$$
|(V-W)(f ; x)| \leq\left\|f^{\prime \prime}\right\| \alpha(x)+\left\|f^{\prime \prime \prime}\right\| \beta(x)+\left\|f^{I V}\right\| \gamma(x),
$$

where

$$
\begin{gathered}
\alpha(x):=\sum_{k \in K}\left|\mu_{2}^{F_{k}}-\mu_{2}^{G_{k}}\right| p_{k}(x), \beta(x):=\sum_{k \in K}\left|\mu_{3}^{F_{k}}-\mu_{3}^{G_{k}}\right| p_{k}(x), \\
\gamma(x):=\sum_{k \in K}\left(\mu_{4}^{F_{k}}+\mu_{4}^{G_{k}}\right) p_{k}(x) .
\end{gathered}
$$

Theorem 3.7. [2] Let $I=[0,1], f \in C[0,1], 0<h \leq \frac{1}{2}, x \in[0,1]$. Then

$$
|(V-W)(f ; x)| \leq \frac{3}{2}\left(1+\frac{\sigma(x)}{h^{2}}\right) \omega_{2}(f, h)+\frac{5 \delta}{h} \omega_{1}(f, h) .
$$

Theorem 3.8. [2] Let $I=[0,1], f \in C[0,1], 0<h<1, x \in[0,1]$ and $b^{F_{k}}=b^{G_{k}}, k \in K$. Then

$$
|(V-W)(f ; x)| \leq c\left[\left(2+\frac{\gamma(x)}{h^{4}}\right) \omega_{4}(f, h)+\frac{\beta(x)}{h^{3}} \omega_{3}(f, h)+\frac{\alpha(x)}{h^{2}} \omega_{2}(f, h)\right],
$$

where $c$ is an absolute constant.

The classical Durrmeyer operators are defined as

$$
M_{n}(f ; x)=(n+1) \sum_{k=0}^{n} p_{n, k}(x) \int_{0}^{1} p_{n, k}(t) f(t) d t, x \in[0,1],
$$

where $p_{n, k}(x)=\left(\begin{array}{l}n \\ k\end{array}\right) x^{k}(1-x)^{n-k}$ and $f$ is an integrable function on $[0,1]$.

Proposition 3.6. [2] For Bernstein operators and Durrmeyer operators the following relations hold:

i) $\left|\left(B_{n}-M_{n}\right)(f ; x)\right| \leq \sigma(x)\left\|f^{\prime \prime}\right\|+\omega_{1}\left(f, \frac{1}{n+2}\right)$, for $f^{\prime \prime} \in C[0,1]$;

ii) $\left|\left(B_{n}-M_{n}\right)(f ; x)\right| \leq 3 \omega_{2}(f, \sqrt{\sigma(x)})+\frac{5}{(n+2) \sqrt{\sigma(x)}} \omega_{1}(f, \sqrt{\sigma(x)})$, for $f \in C[0,1]$, where $\sigma(x)=\frac{1}{2(n+3)(n+2)^{2}}\{x(1-x) n(n-1)+n+1\} \leq \frac{1}{8(n+3)}$.

Proposition 3.7. [2] The Bernstein operators and the genuine Bernstein-Durrmeyer operators verify the following relations

i) $\left|\left(B_{n}-U_{n}\right)(f ; x)\right| \leq \sigma(x)\left\|f^{\prime \prime}\right\|, f^{\prime \prime} \in C[0,1]$,

ii) $\left|\left(B_{n}-U_{n}\right)(f ; x)\right| \leq 3 \omega_{2}(f, \sqrt{\sigma(x)}), f \in C[0,1]$,

$$
\text { where } \sigma(x)=\frac{x(1-x)(n-1)}{2 n(n+1)} \leq \frac{1}{8(n+1)} \text {. }
$$


Proposition 3.8. [2] The composition of two Bernstein operators $D_{n}:=B_{n} \circ B_{n+1}$ and genuine Bernstein-Durrmeyer operators $U_{n}$ verify
i) $\left|\left(D_{n}-U_{n}\right)(f ; x)\right| \leq \frac{n-1}{n(n+1)} x(1-x)\left\|f^{\prime \prime}\right\|, f^{\prime \prime} \in C[0,1]$;
ii) $\left|\left(D_{n}-U_{n}\right)(f ; x)\right| \leq \frac{x(1-x)}{2(n+1)^{2}}\left(\frac{1}{3}\left\|f^{(3)}\right\|+\frac{1}{8}\left\|f^{(4)}\right\|\right), f^{(4)} \in C[0,1]$;
iii) $\left|\left(D_{n}-U_{n}\right)(f ; x)\right| \leq 3 \omega_{2}\left(f, \sqrt{\frac{(n-1) x(1-x)}{n(n+1)}}\right), f \in C[0,1]$;
iv) $\left|\left(D_{n}-U_{n}\right)(f ; x)\right| \leq c\left[\frac{33}{16} \omega_{4}\left(f, \sqrt[4]{\frac{x(1-x)}{(n+1)^{2}}}\right)+\frac{\sqrt[4]{x(1-x)}}{6 \sqrt{n+1}} \omega_{3}\left(f, \sqrt[4]{\frac{x(1-x)}{(n+1)^{2}}}\right)\right], f \in$ $C[0,1]$, where $c$ is an absolute constant and $n \geq 6$.

Let $K_{n}$ be the Kantorovich operators defined in [17] as follows

$$
K_{n}(f ; x)=(n+1) \sum_{k=0}^{n} p_{n, k}(x) \int_{\frac{k}{n+1}}^{\frac{k+1}{n+1}} f(t) d t .
$$

Proposition 3.9. [2] The Bernstein operators and the Kantorovich operators verify the following relations
i) $\left|\left(K_{n}-B_{n}\right)(f ; x)\right| \leq \frac{1}{24(n+1)^{2}}\left\|f^{\prime \prime}\right\|+\omega_{1}\left(f, \frac{1}{2(n+1)}\right), f^{\prime \prime} \in C[0,1]$;
ii) $\left|\left(K_{n}-B_{n}\right)(f ; x)\right| \leq 3 \omega_{2}\left(f, \frac{1}{2 \sqrt{6}(n+1)}\right)+5 \sqrt{6} \omega_{1}\left(f, \frac{1}{2 \sqrt{6}(n+1)}\right), f \in C[0,1]$.

This result can be extended for a generalized class of Kantorovich-type operators. Let $C_{b}[0, \infty)$ be the space of all real-valued continuous functions on $[0, \infty)$ with $\|f\|<\infty$ and $V_{n}: C_{b}[0, \infty) \rightarrow$ $C_{b}[0, \infty), V_{n}(f ; x)=\sum_{k=0}^{\infty} f\left(\frac{k}{n}\right) \varphi_{n, k}(x)$ be a positive linear operator, where $\left(\varphi_{n, k}\right)_{k \geq 0}$ is a sequence of real-valued functions which verify:
i) $\varphi_{n, k}(x) \geq 0, k \geq 0, \quad x \in[0, \infty)$,
ii) $\varphi_{n, k} \in C[0, \infty)$;
iii) $\sum_{k=0}^{\infty} \varphi_{n, k}(x)=1$.

Let $W_{n}: C_{b}[0, \infty) \rightarrow C_{b}[0, \infty)$ be the Kantorovich generalized variant of the operator $V_{n}$. Therefore,

$$
W_{n}(f ; x)=n \sum_{k=0}^{\infty} \varphi_{n, k}(x) \int_{\frac{k}{n}}^{\frac{k+1}{n}} f(t) d t .
$$

Proposition 3.10. [2] With the above notation,

$$
\left|W_{n}(f ; x)-V_{n}(f ; x)\right| \leq \frac{1}{24 n^{2}}\left\|f^{\prime \prime}\right\|+\omega_{1}\left(f, \frac{1}{2 n}\right), f^{(i)} \in C_{b}[0, \infty), i \in\{0,1,2\} .
$$

\section{DIFFERENCES OF $U_{n}^{\rho}$ OPERATORS}

The class of operators $U_{n}^{\rho}$ was introduced in [26] by Păltănea and further investigated by Păltănea and Gonska in [11] and [12]. 
Let $\rho>0$ and $n \in \mathbb{N}$. The operators $U_{n}^{\rho}: C[0,1] \rightarrow \prod_{n}$ are defined by

$$
\begin{aligned}
U_{n}^{\rho}(f ; x) & :=\sum_{k=0}^{n} F_{k}^{\rho}(f) p_{n, k}(x) \\
& :=\sum_{k=1}^{n-1}\left(\int_{0}^{1} \frac{t^{k \rho-1}(1-t)^{(n-k) \rho-1}}{B(k \rho,(n-k) \rho)} f(t) d t\right) p_{n, k}(x)+f(0)(1-x)^{n}+f(1) x^{n},
\end{aligned}
$$

for $f \in C[0,1], x \in[0,1]$.

Remark 4.1. For $\rho=1$ and $f \in C[0,1]$, we obtain the genuine Bernstein-Durrmeyer operators, while for $\rho \rightarrow \infty$, for each $f \in C[0,1]$ the sequence $U_{n}^{\rho}(f ; x)$ converges uniformly to the Bernstein polynomial $B_{n}(f ; x)$.

H. Gonska et al. [12] proved that for $n \geq 1$ and $f \in C[0,1]$,

$$
\lim _{\rho \rightarrow 0^{+}} U_{n}^{\rho} f=B_{1} f, \text { uniformly on }[0,1] .
$$

Moreover, the following result was obtained:

Theorem 4.9. [12] For $U_{n}^{\rho}, 0<\rho<\infty, n \geq 1$, we have

$$
\left|U_{n}^{\rho} f(x)-B_{1} f(x)\right| \leq \frac{9}{4} \omega_{2}\left(f ; \sqrt{\frac{n \rho-\rho}{n \rho+1} x(1-x)}\right) .
$$

The following result was obtained with the method presented in [9].

Proposition 4.11. ([28], [30]) Let $f \in C[0,1], n \geq 1, \rho, r>0, x \in[0,1]$. The following inequality is verified

$$
\begin{aligned}
\left|\left(U_{n}^{\rho}-U_{n}^{r}\right)(f ; x)\right| & \leq c_{1} \omega_{2}\left(f ; \sqrt{\frac{1}{2}\left(U_{n}^{\rho}+U_{n}^{r}\right)\left(\left|e_{1}-x\right|^{2} ; x\right)}\right) \\
& \leq c_{1} \omega_{2}\left(f ; \sqrt{\frac{1}{2} \frac{2 n \rho r+(n+1)(\rho+r)+2}{(n \rho+1)(n r+1)} x(1-x)}\right) .
\end{aligned}
$$

Here $c_{1}$ is an absolute constant independent of $f, x, \rho$ and $r$.

Another result in this direction was obtained in [28] and [30]:

Theorem 4.10. ([28], [30]) Let $f \in C[0,1], n \geq 1, \rho, r>0, x \in[0,1]$. Then

$$
\left|\left(U_{n}^{\rho}-U_{n}^{r}\right)(f ; x)\right| \leq \frac{9}{4} \omega_{2}\left(f ; \sqrt{\frac{(n-1)|\rho-r|}{(n \rho+1)(n r+1)} x(1-x)}\right) .
$$

In the next statement we give some estimates of the difference $U_{n}^{\rho}-U_{n}^{r}$ using the results proved in Section 3:

Proposition 4.12. The following properties hold

i) $\left|\left(U_{n}^{\rho}-U_{n}^{r}\right)(f ; x)\right| \leq \frac{(n-1)[2+(\rho+r) n]}{2 n(1+\rho n)(1+r n)} x(1-x)\left\|f^{\prime \prime}\right\|, f^{\prime \prime} \in C[0,1]$; 
ii) $\left|\left(U_{n}^{\rho}-U_{n}^{r}\right)(f ; x)\right| \leq \frac{(n-1)|r-\rho|}{2(1+\rho n)(1+r n)} x(1-x)\left\|f^{\prime \prime}\right\|$

$$
\begin{aligned}
& +\frac{1}{3} x(1-x)(n-1) \frac{|r-\rho|[3+n(r+\rho)]}{(1+\rho n)(2+\rho n)(1+r n)(2+r n)}\left\|f^{\prime \prime \prime}\right\| \\
& +\frac{1}{32} x(1-x) \frac{n^{2}\left(\rho^{2}+r^{2}\right)+4 n(\rho+r)+6}{(1+\rho n)(3+\rho n)(1+r n)(3+r n)}\left\|f^{I V}\right\|, f^{(4)} \in C[0,1], \\
& n \rho \geq 6, \quad n r \geq 6 ;
\end{aligned}
$$

iii) $\left|\left(U_{n}^{\rho}-U_{n}^{r}\right)(f ; x)\right| \leq 3 \omega_{2}\left(f, \sqrt{\frac{2+(\rho+r) n}{2(1+\rho n)(1+r n)} x(1-x)}\right), f \in C[0,1]$.

Denote by $[A ; B]:=A B-B A$ the commutator of two positive linear operators $A$ and $B$. In [28] the following result concerning the comutator $\left[U_{n}^{\varrho} ; U_{n}^{\sigma}\right]$ was obtained:

Theorem 4.11. [28] For each $f \in C^{6}[0,1]$ one has

$$
\lim _{n \rightarrow \infty} n^{3}\left(U_{n}^{\rho} U_{n}^{r}-U_{n}^{r} U_{n}^{\rho}\right) f(x)=\frac{(r-\rho)(\rho+1)(r+1)}{\rho^{2} r^{2}} x(1-x) f^{(4)}(x),
$$

uniformly with respect to $x \in[0,1]$.

\section{DIFFERENCES OF LINEAR POSITIVE OPERATORS DEFINED ON UNBOUNDED INTERVAL}

Most of the results presented in the previuos sections are given for operators defined over bounded intervals. Very recently, Aral et al.[4] obtained some quantitative results in terms of weighted modulus of continuity for differences of certain positive linear operators defined on unbounded intervals.

Let $C[0, \infty)$ be the set of all continuous functions $f$ defined on $[0, \infty)$ and $B_{2}[0, \infty)$ the set of all functions $f$ defined on $[0, \infty)$ satisfying the condition $|f(x)| \leq M\left(1+x^{2}\right)$ for some positive constant $M$, which may depend only on $f . C_{2}[0, \infty)$ denotes the subspace of all continuous functions in $B_{2}[0, \infty)$. Let $C_{2}^{*}[0, \infty)$ be the closed subspace of $C_{2}[0, \infty)$ formed by the functions $f$ for which the limit $\lim _{x \rightarrow \infty} \frac{f(x)}{1+x^{2}}$ exists and is finite. $B_{2}[0, \infty)$ is a linear normed space with the norm $\|f\|_{2}=\sup _{x>0} \frac{|f(x)|}{1+x^{2}}$.

In 2001, Ispir [16] introduced the weighted modulus of continuity as follows:

$$
\Omega(f ; \delta)=\sup _{|h|<\delta, x \in[0, \infty)} \frac{|f(x+h)-f(x)|}{\left(1+h^{2}\right)\left(1+x^{2}\right)} \text {, for } f \in C_{2}[0, \infty) .
$$

Let $e_{i}(x)=x^{i}, x \in[0, \infty), i \in \mathbb{N}$ and $F: D \rightarrow \mathbb{R}$ be a positive linear functional defined on a linear subspace $D$ of $C[0, \infty)$ which contains $C_{2}[0, \infty)$ and the polynomials up to degree 6 , such that $F\left(e_{0}\right)=1, b^{F}:=F\left(e_{1}\right)$ and

$$
\mu_{i}^{F}=F\left(e_{1}-b^{F} e_{0}\right)^{i}, i \in \mathbb{N}, 0 \leq i \leq 6 .
$$

Therefore, $\mu_{0}^{F}=1, \mu_{1}^{F}=0$ and $\mu_{2}^{F}=F\left(e_{2}\right)-\left(b^{F}\right)^{2} \geq 0$.

The next estimate concerning the functional $F$ was given in [4].

Lemma 5.3. [4] Let $f \in C_{2}[0, \infty)$ with $f^{\prime \prime} \in C_{2}^{*}[0, \infty)$ and $0<\delta \leq 1$. Then we have

$$
\left|F(f)-f\left(b^{F}\right)\right| \leq \frac{1}{2}\left|f^{\prime \prime}\left(b^{F}\right)\right| \mu_{2}^{F}+8\left(1+\left(b^{F}\right)^{2}\right) \Omega\left(f^{\prime \prime} ; \delta\right)\left(\mu_{2}^{F}+\frac{\mu_{6}^{F}}{\delta^{4}}\right) .
$$


Let $\mathbb{K}$ be a set of non-negative integers and consider $p_{k}:[0, \infty) \rightarrow[0, \infty), k \in \mathbb{K}$. Denote

$$
U(f ; x)=\sum_{k \in \mathbb{K}} F_{k}(f) p_{k}(x) \text { and } V(f ; x)=\sum_{k \in \mathbb{K}} G_{k}(f) p_{k}(x),
$$

where $U, V: D \rightarrow B_{2}[0, \infty)$ and $F_{k}, G_{k}: D \rightarrow \mathbb{R}$ are positive linear functionals such that $F_{k}\left(e_{0}\right)=1$ and $G_{k}\left(e_{0}\right)=1$. Applying Lemma 5.3 for the functionals $F_{k}$ and $G_{k}$ the following result was obtained by Aral et al. [4]:

Theorem 5.12. [4] Let $f \in C_{2}[0, \infty)$ with $f^{\prime \prime} \in C_{2}^{*}[0, \infty)$. Then

$$
|(U-V)(f ; x)| \leq \frac{1}{2}\left\|f^{\prime \prime}\right\|_{2} \beta(x)+8 \Omega\left(f^{\prime \prime} ; \delta_{1}\right)(1+\beta(x))+16 \Omega\left(f ; \delta_{2}\right)(\gamma(x)+1),
$$

where

$$
\begin{aligned}
& \beta(x)=\sum_{k \in \mathbb{K}} p_{k}(x)\left\{\left[1+\left(b^{F_{k}}\right)^{2}\right] \mu_{2}^{F_{k}}+\left[1+\left(b^{G_{k}}\right)^{2}\right] \mu_{2}^{G_{k}}\right\}, \\
& \gamma(x)=\sum_{k \in \mathbb{K}} p_{k}(x)\left[1+\left(b^{F_{k}}\right)^{2}\right], \\
& \delta_{1}(x)=\left\{\sum_{k \in \mathbb{K}} p_{k}(x)\left\{\left[1+\left(b^{F_{k}}\right)^{2}\right] \mu_{6}^{F_{k}}+\left[1+\left(b^{G_{k}}\right)^{2}\right] \mu_{6}^{G_{k}}\right\}\right\}^{1 / 4}, \\
& \delta_{2}(x)=\left\{\sum_{k \in \mathbb{K}} p_{k}(x)\left(1+\left(b^{F_{k}}\right)^{2}\right)\left(b^{F_{k}}-b^{G_{k}}\right)^{4}\right\}^{1 / 4},
\end{aligned}
$$

and we suppose that $\delta_{1}(x) \leq 1, \delta_{2}(x) \leq 1$.

In [4] this result was applied for the sequences of Szász-Mirakyan [31] and Szász-MirakyanDurrmeyer operators [23] defined as

$$
\begin{gathered}
\mathbb{S}_{n}(f ; x)=\sum_{k=0}^{\infty} f\left(\frac{k}{n}\right) s_{n, k}(x), \\
\mathbb{D}_{n}(f ; x)=n \sum_{k=0}^{\infty} s_{n, k}(x) \int_{0}^{\infty} f(t) s_{n, k}(t) d t, x \in[0, \infty),
\end{gathered}
$$

where $s_{n, k}(x)=e^{-n x} \frac{(n x)^{k}}{k !}$.

Proposition 5.13. [4] Let $f \in C_{2}[0, \infty)$ with $f^{\prime \prime} \in C_{2}^{*}[0, \infty)$ and $x \geq 0$ is given. Then, for each $n \geq 1$ such that

$$
\begin{aligned}
\delta_{1}^{4}(x) & :=\frac{2}{2 n^{8}}\left(3 n^{5} x^{5}+71 n^{4} x^{4}+\left(3 n^{4}+479 n^{3}\right) x^{3}+\left(44 n^{4}+1064 n^{2}\right) x^{2}\right. \\
& \left.+\left(123 n^{3}+651 n\right) x+53 n^{2}+53\right) \leq 1, \\
\delta_{2}^{4}(x) & :=\frac{x(n x+1)+n}{n^{5}} \leq 1,
\end{aligned}
$$

we have

$$
\left|\left(\mathbb{S}_{n}-\mathbb{D}_{n}\right)(f ; x)\right| \leq \frac{1}{2}\left\|f^{\prime \prime}\right\|_{2} \beta(x)+8 \Omega\left(f^{\prime \prime} ; \delta_{1}(x)\right)(1+\beta(x))+16 \Omega\left(f ; \delta_{2}(x)\right)\left(\frac{x(n x+1)+n}{n}\right),
$$

where $\beta(x)=\frac{n^{3} x^{3}+6 n^{2} x^{2}+n^{3} x+7 n x+n^{2}}{n^{4}}$. 


\section{ESTIMATES FOR THE DIFFERENCES OF POSITIVE LINEAR OPERATORS AND THEIR} DERIVATIVES

It is well-known that de la Valleee-Poussin operator commutes with the derivative. Certainly, this property is not available for the Bernstein operators $B_{n}$. This remark motivated us to estimate in terms of moduli of continuity the differences $\left(L_{n} f\right)^{(k)}-L_{n-k}\left(f^{(k)}\right)$ for certain positive linear operators. In the following we will exemplify for Bernstein operators and Durrmeyer operators.

Theorem 6.13. [3] For Bernstein operators the following property holds:

$$
\left\|\left(B_{n} f\right)^{(r)}-B_{n-r}\left(f^{(r)}\right)\right\| \leq \frac{(r-1) r}{2 n}\left\|f^{(r)}\right\|+\omega\left(f^{(r)}, \frac{r}{n}\right), f \in C^{r}[0,1], r=0,1, \ldots n .
$$

Theorem 6.14. [3] The Kantorovich operators verify

$$
\left\|\left(K_{n} f\right)^{(r)}-K_{n-r}\left(f^{(r)}\right)\right\| \leq \frac{(r+1) r}{2(n+1)}\left\|f^{(r)}\right\|+\omega\left(f^{(r)}, \frac{r+1}{n+1}\right), f \in C^{r}[0,1], r=0,1, \ldots n .
$$

7. DISCRETE OPERATORS ASSOCIATED WITH CERTAIN INTEGRAL OPERATORS

In 2011, I. Raşa [27] constructed discrete operators associated with certain integral operators using a probabilistic approach.

Let $I_{n}: C[a, b] \rightarrow C[a, b], n \geq 1$, be a sequence of positive linear operators of the form

$$
I_{n}(f ; x)=\sum_{k=0}^{n} h_{n, k}(x) A_{n, k}(f), f \in C[a, b], x \in[a, b],
$$

where $h_{n, k} \in C[a, b], h_{n, k} \geq 0$ and

$$
A_{n, k}(f)=\int_{a}^{b} f(t) d \mu_{n, k}(t),
$$

with $\mu_{n, k}$ probability Borel measures on $[a, b], n \geq 1, k=0,1, \ldots, n$.

Raşa [27] introduced the following discrete operator associated with the sequence $\left(I_{n}\right)$

$$
D_{n}(f ; x)=\sum_{k=0}^{n} h_{n, k}(x) f\left(x_{n, k}\right),
$$

where $x_{n, k}=\int_{a}^{b} t d \mu_{n, k}(t)$.

For example, the associated operators of the genuine Bernstein-Durrmeyer operators are Bernstein operators.

D. Mache intoduced the sequence of positive linear operators (see [21], [22])

$$
P_{n}(f ; x):=\sum_{k=0}^{n} p_{n, k}(x) T_{n, k}(f), n \geq 1,
$$

where

$$
T_{n, k}(f):=\frac{\int_{0}^{1} f(t) t^{c k+a}(1-t)^{c(n-k)+b} d t}{B(c k+a+1, c(n-k)+b+1)},
$$

for $a, b>-1, \alpha \geq 0, c:=c_{n}:=\left[n^{\alpha}\right]$ and $B$ is the Beta function.

Remark 7.2. [27] The sequence of positive linear operators $\left(P_{n}\right)$ represents a link between the Durrmeyer operators with Jacobi weights $(\alpha=0)$ and the Bernstein operators $(\alpha \rightarrow \infty)$. 
The sequence of positive linear operators $\left(V_{n}\right)$ defined as follows

$$
V_{n}(f ; x):=\sum_{k=0}^{n} p_{n, k}(x) f\left(\frac{c k+a+1}{c n+a+b+2}\right), f \in C[0,1], x \in[0,1]
$$

is associated with the sequence $\left(P_{n}\right)$ (see [27]).

Remark 7.3. [27] For $a=b=-1$, or $\alpha \rightarrow \infty$, we get the classical Bernstein operators. For $\alpha=0$, the operators $V_{n}$ reduce to the operators considered by D.D. Stancu in [29].

Theorem 7.15. [27] For $n \geq 1, x \in[0,1]$, and $f \in C^{2}[0,1]$ we have

$$
\begin{gathered}
\left|P_{n}(f ; x)-V_{n}(f ; x)\right| \leq \\
\frac{c^{2} n(n-1) x(1-x)+c n(b-a) x+c n(a+1)+(a+1)(b+1)}{2(c n+a+b+2)^{2}(c n+a+b+3)}\left\|f^{\prime \prime}\right\| .
\end{gathered}
$$

The study on this topic was continued by Heilmann et al. [15]. They associated to an integral operator a discrete one which is conceptually simpler, and study the relations between them.

Let $I \subset \mathbb{R}$ be an interval, $H$ a subspace of $C(I)$ containing $e_{i}, i=0,1,2$ and $L: H \rightarrow C(I)$ be a positive linear operator such that $L e_{0}=e_{0}$. For $f \in H$ and $A_{j}: H \rightarrow \mathbb{R}$ positive linear functionals such that $A_{j}\left(e_{0}\right)=1$ and $p_{j} \in C(I), p_{j} \geq 0, \sum_{j=0}^{\infty} p_{j}=e_{0}$, the following operator was defined in [15]:

$$
L f:=\sum_{j=0}^{\infty} A_{j}(f) p_{j} .
$$

The discrete operator associated with $L$ is defined by

$$
D: H \rightarrow C(I), D f:=\sum_{j=0}^{\infty} f\left(b_{j}\right) p_{j},
$$

where $b_{j}:=A_{j}\left(e_{1}\right)$. The point evaluation functional at $b_{j}$ is conceptually simpler than $A_{j}$. So, from this point of view, $D$ is simpler than $L$.

Let $\operatorname{Var} A_{j}:=A_{j}\left(e_{2}\right)-b_{j}^{2}, j \geq 0$. $\operatorname{Var} A_{j}$ shows how far is $A_{j}$ from the point evaluation at $b_{j}$. Define by

$$
E(L)(x):=\sum_{j=0}^{\infty}\left(\operatorname{Var} A_{j}\right) p_{j}(x), x \in I .
$$

The following relation between $L$ and $D$ was obtained in [15]:

$$
|L f(x)-D f(x)| \leq \frac{1}{2}\left\|f^{\prime \prime}\right\|_{\infty} E(L)(x), x \in I .
$$

In the above relation $E(L)(x)$ shows how far is $L$ from $D$.

The discrete operators associated with Baskakov type operators, genuine Baskakov-Durrmeyer type operators, and their Kantorovich modifications were constructed and the above general result was applied in this context. 


\section{DIFFERENCES OF INVERSES OF POSITIVE LINEAR OPERATORS}

The Voronovskaya type result for the Bernstein operators is well known:

$$
\lim _{n \rightarrow \infty} n\left(B_{n}(f ; x)-f(x)\right)=\frac{x(1-x)}{2} f^{(2)}(x), f \in C^{2}[0,1] .
$$

Moreover, Abel and Ivan [1] obtained the following result

$$
\begin{aligned}
& \lim _{n \rightarrow \infty} n\left[n\left(B_{n}(f ; x)-f(x)\right)-\frac{x(1-x)}{2} f^{(2)}(x)\right] \\
& =\frac{x(1-x)}{24}\left(3 x(1-x) f^{(4)}(x)+4(1-2 x) f^{(3)}(x)\right), f \in C^{4}[0,1] .
\end{aligned}
$$

Similarly, Voronovskaya type results for Beta operators were proved by Gonska et al. [7] as follows

$$
\begin{aligned}
& \lim _{n \rightarrow \infty} n\left(\overline{\mathbb{B}}_{n}(f ; x)-f(x)\right)=\frac{x(1-x)}{2} f^{(2)}(x), f \in C^{2}[0,1] \\
& \lim _{n \rightarrow \infty} n\left[n\left(\overline{\mathbb{B}}_{n}(f ; x)-f(x)\right)-\frac{x(1-x)}{2} f^{(2)}(x)\right] \\
& \quad=\frac{x(1-x)}{24}\left(3 x(1-x) f^{(4)}(x)+8(1-2 x) f^{(3)}(x)-12 f^{(2)}(x)\right), f \in C^{4}[0,1] .
\end{aligned}
$$

Voronovskaja type formulas are usually established for sequences of positive linear operators. Nasaireh et al. [24] obtained some general formulas concerning compositions of operators on Banach spaces, without any assumption of positivity. Let $X$ be a Banach space and $W \subset Z \subset Y$ linear subspaces of $X$. Let $A, B: Y \rightarrow X ; U, V: Z \rightarrow X$ be linear operators. Consider also two sequences of linear operators $P_{n}: X \rightarrow X, Q_{n}: Y \rightarrow X, n \geq 1$, and suppose that each $P_{n}$ is bounded.

Theorem 8.16. [24] (i) Suppose that

$$
\lim _{n \rightarrow \infty} P_{n} x=x, x \in X
$$

and

$$
\lim _{n \rightarrow \infty} n\left(P_{n} y-y\right)=A y, \quad \lim _{n \rightarrow \infty} n\left(Q_{n} y-y\right)=B y, y \in Y .
$$

Then

$$
\lim _{n \rightarrow \infty} n\left(P_{n} Q_{n} y-y\right)=A y+B y, y \in Y .
$$

ii) In addition to (8.11) and (8.12), suppose that

$$
\begin{gathered}
B z \in Y, z \in Z, \\
\lim _{n \rightarrow \infty} n\left[n\left(P_{n} z-z\right)-A z\right]=U z ; \lim _{n \rightarrow \infty} n\left[n\left(Q_{n} z-z\right)-B z\right]=V z, z \in Z .
\end{gathered}
$$

Then

$$
\lim _{n \rightarrow \infty} n\left[n\left(P_{n} Q_{n} z-z\right)-A z-B z\right]=U z+V z+A B z, z \in Z .
$$

Very recently, this result was extended for a more general case by Heilmann et al. [14]. 
Using Theorem 8.16, Voronovskaya type results for genuine Bernstein-Durrmeyer operators were proved by Nasaireh et al. [24] as follows

$$
\begin{aligned}
& \lim _{n \rightarrow \infty} n\left(U_{n}(f ; x)-f(x)\right)=x(1-x) f^{(2)}(x), f \in C^{2}[0,1] \\
& \lim _{n \rightarrow \infty} n\left[n\left(U_{n}(f ; x)-f(x)\right)-x(1-x) f^{(2)}(x)\right] \\
& \quad=\frac{x(1-x)}{2}\left(x(1-x) f^{(4)}(x)+2(1-2 x) f^{(3)}(x)-2 f^{(2)}(x)\right), f \in C^{4}[0,1] .
\end{aligned}
$$

Let $\Pi_{n}$ be the space of all polynomial functions of degree at most $n$, defined on $\mathbb{R}$, and $\Pi=\cup_{n \geq 0} \Pi_{n}$. Nasaireh et al. [24] expressed the inverse of Beta operator as follows:

$$
\overline{\mathbb{B}}_{n}^{-1}: \Pi \rightarrow \Pi, n \geq 1, \quad \overline{\mathbb{B}}_{n}^{-1}(p ; x)=\sum_{k=0}^{m} \frac{(n)_{k}}{n^{k}}\left[0,-\frac{1}{n}, \ldots,-\frac{k}{n} ; p\right] x^{k}, p \in \Pi_{m} .
$$

Using Theorem 8.16 the following Voronovskaya type results for inverses of Beta operators, Bernstein operators and genuine Bernstein-Durrmeyer operators can be obtained (see [24, 25])

Theorem 8.17. Let $m \geq 0$ and $p_{n} \in \Pi_{m}, n \geq 1$. Suppose that the sequence $\left(p_{n}\right)$ is uniformly convergent on $[0,1]$ to $p \in \Pi_{m}$. Then

$$
\begin{aligned}
\text { i) } & \lim _{n \rightarrow \infty} n\left(\overline{\mathbb{B}}_{n}^{-1}\left(p_{n} ; x\right)-p_{n}(x)\right)=-\frac{x(1-x)}{2} p^{\prime \prime}(x), \\
\text { ii) } & \lim _{n \rightarrow \infty} n\left[n\left(\overline{\mathbb{B}}_{n}^{-1}\left(p_{n} ; x\right)-p_{n}(x)\right)+\frac{x(1-x)}{2} p_{n}^{\prime \prime}(t)\right] \\
= & \frac{x(1-x)}{24}\left\{3 x(1-x) p^{(4)}(x)+4(1-2 x) p^{(3)}(x)\right\} .
\end{aligned}
$$

Theorem 8.18. Let $m \geq 0$ and $p_{n} \in \Pi_{m}, n \geq 1$. Suppose that the sequence $\left(p_{n}\right)$ is uniformly convergent on $[0,1]$ to $p \in \Pi_{m}$. Then
i) $\lim _{n \rightarrow \infty} n\left(B_{n}^{-1}\left(p_{n} ; x\right)-p_{n}(x)\right)=-\frac{x(1-x)}{2} p^{\prime \prime}(x)$,
ii) $\lim _{n \rightarrow \infty} n\left[n\left(B_{n}^{-1}\left(p_{n} ; x\right)-p_{n}(x)\right)+\frac{x(1-x)}{2} p_{n}^{\prime \prime}(t)\right]$$$
=\frac{x(1-x)}{24}\left\{3 x(1-x) p^{(4)}(x)+8(1-2 x) p^{(3)}(x)-12 p^{\prime \prime}(x)\right\} .
$$

Theorem 8.19. Let $m \geq 0$ and $p_{n} \in \Pi_{m}, n \geq 1$. Suppose that the sequence $\left(p_{n}\right)$ is uniformly convergent on $[0,1]$ to $p \in \Pi_{m}$. Then

$$
\text { i) } \begin{aligned}
& \lim _{n \rightarrow \infty} n\left(U_{n}^{-1}\left(p_{n} ; x\right)-p_{n}(x)\right)=-x(1-x) p^{\prime \prime}(x), \\
\text { ii) } & \lim _{n \rightarrow \infty} n\left[n\left(U_{n}^{-1}\left(p_{n} ; x\right)-p_{n}(x)\right)+x(1-x) p_{n}^{\prime \prime}(x)\right] \\
= & \frac{x(1-x)}{2}\left[x(1-x) p^{(4)}(x)+2(1-2 x) p^{(3)}(x)-2 p^{\prime \prime}(x)\right] .
\end{aligned}
$$

From the above results one can obtain new estimates concerning certain operators and their inverses.

Proposition 8.14. Let $p \in \Pi$. Then

$$
\lim _{n \rightarrow \infty} n\left(B_{n}(p ; x)-B_{n}^{-1}(p ; x)\right)=x(1-x) p^{\prime \prime}(x) .
$$

Proposition 8.15. Let $p \in \Pi$. Then

$$
\lim _{n \rightarrow \infty} n\left[n\left(B_{n}(p ; x)-B_{n}^{-1}(p ; x)\right)-x(1-x) p^{\prime \prime}(x)\right]=\frac{x(1-x)}{6}\left[3 p^{\prime \prime}(x)-(1-2 x) p^{(3)}(x)\right] .
$$


Proposition 8.16. Let $p \in \Pi$. Then

$$
\lim _{n \rightarrow \infty} n\left(\overline{\mathbb{B}}_{n}(p ; x)-\overline{\mathbb{B}}_{n}^{-1}(p ; x)\right)=x(1-x) p^{\prime \prime}(x) .
$$

Proposition 8.17. Let $p \in \Pi$. Then

$$
\lim _{n \rightarrow \infty} n\left[n\left(\overline{\mathbb{B}}_{n}(p ; x)-\overline{\mathbb{B}}_{n}^{-1}(p ; x)\right)-x(1-x) p^{\prime \prime}(x)\right]=\frac{x(1-x)}{6}\left[(1-2 x) p^{(3)}(x)-3 p^{(2)}(x)\right] .
$$

Proposition 8.18. Let $p \in \Pi$. Then

$$
\lim _{n \rightarrow \infty} n\left[U_{n}(p ; x)-U_{n}^{-1}(p ; x)\right]=2 x(1-x) p^{\prime \prime}(x) .
$$

Proposition 8.19. Let $p \in \Pi$. Then

$$
\lim _{n \rightarrow \infty} n\left[n\left(U_{n}(p ; x)-U_{n}^{-1}(p ; x)\right)-2 x(1-x) p^{\prime \prime}(x)\right]=0 .
$$

Proposition 8.20. Let $p \in \Pi$. Then

$$
\lim _{n \rightarrow \infty} n^{2}\left(\overline{\mathbb{B}}_{n}^{-1}(p ; x)-B_{n}^{-1}(p ; x)\right)=\frac{x(1-x)}{6}\left\{3 p^{\prime \prime}(x)-(1-2 x) p^{(3)}(x)\right\} .
$$

Other results of this type can be obtained in a similar way.

\section{REFERENCES}

[1] U. Abel, M. Ivan, Asymptotic expansion of the multivariate Bernstein polynomials on a simplex, Approx. Theory Appl. 16 (2000), 85-93.

[2] A. M. Acu, I. Raşa, New estimates for the differences of positive linear operators, Numerical Algorithms 73(3) (2016), 775-789.

[3] A. M. Acu, I. Raşa, Estimates for the differences of positive linear operators and their derivatives, arXiv:1810.08839v1, submitted.

[4] A. Aral, D. Inoan and I. Raşa, On differences of linear positive operators, Anal. Math. Phys.(2018). DOI https://doi.org/10.1007/s1332

[5] S. N. Bernstein, Démonstration du théorème de Weierstrass fondée sur le calcul des probabilités, Communications de la Société Mathematique de Kharkov, 13 (1913), 1-2.

[6] W. Chen, On the modified Bernstein-Durrmeyer operator, Report of the Fifth Chinese Conference on Approximation Theory, Zhen Zhou, China, 1987.

[7] H. Gonska, I. Raşa, E.-D. Stănilă, Beta operators with Jacobi weights, In: Constructive theory of functions, Sozopol 2013 (K. Ivanov, G. Nikolov and R. Uluchev, Eds.), 99-112. Prof. Marin Drinov Academic Publishing House, Sofia, 2014.

[8] H. Gonska, I. Raşa, Differences of positive linear operators and the second order modulus, Carpathian J. Math. 24(3) (2008), 332-340.

[9] H. Gonska, P. Piţul, I. Raşa, On differences of positive linear operators, Carpathian J. Math. 22(1-2) (2006), 65-78.

[10] H. Gonska, P. Piţul, I. Raşa, On Peano's form of the Taylor remainder, Voronovskaja's theorem and the commutator of positive linear operators, in Numerical Analysis and Approximation Theory (Proc. Int. Conf. Cluj-Napoca 2006; ed. by O. Agratini and P. Blaga), Cluj-Napoca, Casa Cărţii de Ştiinţă, 2006, 55-80.

[11] H. Gonska, R. Păltănea, Simultaneous approximation by a class of Bernstein-Durrmeyer operators preserving linear functions, Czechoslovak Math. J. 60(135) (2010), 783-799.

[12] H. Gonska, R. Păltănea, Quantitative convergence theorems for a class of Bernstein-Durrmeyer operators preserving linear functions, Ukrainian Math. J. 62 (2010), 913-922.

[13] T. N. T. Goodman, A. Sharma, A modified Bernstein-Schoenberg operator, Proc. of the Conference on Constructive Theory of Functions, Varna 1987 (ed. by Bl. Sendov et al.). Sofia: Publ. House Bulg. Acad. of Sci., 1988, 166-173.

[14] M. Heilmann, F. Nasaireh, I. Raşa, Complements to Voronovskaja's formula, Chapter 11 In: D. Ghosh et al. (eds.), Mathematics and Computing, Springer Proceedings in Mathematics \& Statistics 253, Springer Nature Singapore Pte Ltd. 2018, https:/ / doi.org/10.1007/978-981-13-2095-8 11.

[15] M. Heilmann, F. Nasaireh, I. Raşa, Discrete Operators associated with Linking Operators, arXiv:1808.07239v1.

[16] N. Ispir, On modified Baskakov operators on weighted spaces Turk. J. Math. 26(3) (2001), 355-365.

[17] L.V. Kantorovich, Sur certain développements suivant les polynômes de la forme de S. Bernstein, I, II, C.R. Acad. URSS 563-568 (1930), 595-600. 
[18] A. Lupaş, The approximation by means of some linear positive operators, in Approximation Theory (M.W. Müller et al., eds), Akademie-Verlag, Berlin, 1995, 201-227.

[19] A. Lupaş, Die Folge der Betaoperatoren, Dissertation, Universität Stuttgart, 1972.

[20] L. Lupaş, A. Lupaş, Polynomials of Binomial Type and Approximation Operators, Studia Univ. Babeş-Bolyai, Mathematica, XXXII, 4 (1987), 61-70.

[21] D.H. Mache, Gewichtete Simultanapproximation in der Lp-Metrik durch das Verfahren der Kantorovic Operatoren, Dissertation, Univ. Dortmund, 1991.

[22] D.H. Mache, A link between Bernstein polynomials and Durrmeyer polynomials with Jacobi weights, In: Approx. Theory VIII, Vol. 1: Approximation and Interpolation, Ch.K. Chui and L.L. Schmaker (Eds.), 403-410, World Scientific Publ., 1995.

[23] S.M. Mazhar, V. Totik, Approximation by modified Szász operators, Acta Sci. Math. 49 (1985), 257-269.

[24] F. Nasaireh, I. Raşa, Another look at Voronovskaya type formulas, Journal of Mathematical Inequalities, 12(1) (2018), 95-105.

[25] F. Nasaireh, Voronovskaja-type formulas and applications, General Mathematics, 25(1-2) (2017), $37-43$.

[26] R. Păltănea, A class of Durrmeyer type operators preserving linear functions, Ann. Tiberiu Popoviciu Sem. Funct. Equat. Approxim. Convex. (Cluj-Napoca) 5 (2007), 109-117.

[27] I. Raşa, Discrete operators associated with certain integral operators, Stud. Univ. Babeş- Bolyai Math., 56(2) (2011), 537-544.

[28] I. Raşa, E. Stănilă, On some operators linking the Bernstein and the genuine Bernstein-Durrmeyer operators, J. Appl. Funct. Anal. 9 (2014), 369-378.

[29] D.D. Stancu, Asupra unei generalizari a polinoamelor lui Bernstein, Stud. Univ. Babes-Bolyai Math. 14 (1969), $31-45$.

[30] E.D. Stănilă, On Bernstein-Euler-Jacobi Operators, Ph D Thesis, Duisburg-Essen University, July, 2014.

[31] O. Szász, Generalization of S. Bernstein's polynomials to the infinite interval J. Res. Natl. Bur. Stand. 45 (1950), $239-245$.

LUCIAN BLAGA UNIVERSITY OF SIBIU

DEPARTMENT OF MATHEMATICS AND INFORMATICS

STR. DR. I. RATIU, NO.5-7, RO-550012

SIBIU, ROMANIA

E-mail address: anamaria.acu@ulbsibiu.ro

TECHNICAL UNIVERSITY OF CLUJ-NAPOCA

FACULTY OF AUTOMATION AND COMPUTER SCIENCE

Department of Mathematics, Str. Memorandumului NR. 28

CluJ-NAPOCA, ROMANIA

E-mail address: hodissever@gmail.com

TECHNICAL UNIVERSITY OF CLUJ-NAPOCA

FACULTY OF AUTOMATION AND COMPUTER SCIENCE

Department of MATHEMATICS, STR. Memorandumului NR. 28

CLUJ-NAPOCA, ROMANIA

E-mail address: ioan.rasa@math.utcluj.ro 